\title{
Sensorless adaptive optics for isoSTED nanoscopy
}

\author{
Jacopo Antonello ${ }^{\mathrm{a}}$, Xiang $\mathrm{Hao}^{\mathrm{b}}$, Edward S. Allgeyer ${ }^{\mathrm{c}}$, Joerg Bewersdorf ${ }^{\mathrm{b}, \mathrm{d}}$, Jens Rittscher ${ }^{\mathrm{e}, \mathrm{f}}$, \\ and Martin J. Booth ${ }^{\text {a,f }}$ \\ ${ }^{a}$ Centre for Neural Circuits and Behaviour, University of Oxford, Oxford OX1 3SR, UK \\ ${ }^{b}$ Department of Cell Biology, Yale School of Medicine, New Haven, Connecticut 06520, USA \\ 'The Gurdon Institute, University of Cambridge, Cambridge CB2 1QN, UK \\ ${ }^{\mathrm{d}}$ Department of Biomedical Engineering, Yale University, New Haven, Connecticut 06510, USA \\ eThe Institute of Biomedical Engineering, Old Road Campus Research Building, Oxford OX3 \\ $7 \mathrm{DQ}, \mathrm{UK}$ \\ ${ }^{\mathrm{f}}$ Department of Engineering Science, University of Oxford, Oxford OX1 3PJ, UK
}

\begin{abstract}
The presence of aberrations is a major concern when using fluorescence microscopy to image deep inside tissue. Aberrations due to refractive index mismatch and heterogeneity of the specimen under investigation cause severe reduction in the amount of fluorescence emission that is collected by the microscope. Furthermore, aberrations adversely affect the resolution, leading to loss of fine detail in the acquired images. These phenomena are particularly troublesome for super-resolution microscopy techniques such as isotropic stimulated-emission-depletion microscopy (isoSTED), which relies on accurate control of the shape and co-alignment of multiple excitation and depletion foci to operate as expected and to achieve the super-resolution effect.

Aberrations can be suppressed by implementing sensorless adaptive optics techniques, whereby aberration correction is achieved by maximising a certain image quality metric. In confocal microscopy for example, one can employ the total image brightness as an image quality metric. Aberration correction is subsequently achieved by iteratively changing the settings of a wavefront corrector device until the metric is maximised. This simplistic approach has limited applicability to isoSTED microscopy where, due to the complex interplay between the excitation and depletion foci, maximising the total image brightness can lead to introducing aberrations in the depletion foci. In this work we first consider the effects that different aberration modes have on isoSTED microscopes. We then propose an iterative, wavelet-based aberration correction algorithm and evaluate its benefits.
\end{abstract}

Keywords: Adaptive optics, wavefront sensing, super-resolution

\section{INTRODUCTION}

Super-resolution techniques enable fluorescence microscopy observations at resolutions that are beyond the diffraction limit of visible light. ${ }^{1}$ Among such techniques is stimulated-emission-depletion (STED) microscopy, ${ }^{2}$ where the super-resolution effect is obtained by superimposing a ring-shaped depletion focus onto a conventional excitation focus. The depletion focus allows fluorescence emission at the centre of the excitation but inhibits emission at the periphery, thus enhancing lateral resolution within the focal plane. Enhancement of the resolution along the optical axis, instead, is best achieved when employing $4 \mathrm{Pi}$ super-resolution microscopes. ${ }^{3-7}$ In this case the axial enhancement is obtained by simultaneously using two microscope objectives that are arranged along a single optical axis and are oriented in opposite directions, such that they have a common focus. ${ }^{2}$ In this work we consider isoSTED microscopy, ${ }^{3}$ which combines STED with the $4 \mathrm{Pi}$ arrangement to achieve quasi-isotropic resolution enhancement.

In conventional, single-objective STED microscopy the resolution enhancement effect is conditional on maintaining accurate control of the shape and alignment of the excitation and depletion foci. In isoSTED microscopy additional requirements arise, since one also needs to maintain optimal alignment between the foci generated via

Further author information: (Send correspondence to martin.booth@eng.ox.ac.uk) 
the two objectives, and to control the resulting interference pattern within the focal volume. It is well-known, ${ }^{8-11}$ however, that such stringent requirements are invalidated in the presence of aberrations. Aberrations may arise due to refractive-index mismatches ${ }^{12}$ or, more commonly, they are caused by variations in the index of refraction within specimens. ${ }^{13}$ As a consequence, when imaging deep inside tissue, one needs to implement adaptive optics techniques ${ }^{13}$ in order to guarantee that super-resolution is indeed attained.

Adaptive optics has been previously demonstrated in single-objective STED. ${ }^{14-16}$ When a spatial light modulator (SLM) is used to create the $2 \mathrm{D}$ or $3 \mathrm{D}$ depletion focus, one may easily apply aberration correction to the depletion beam only using the same SLM. ${ }^{14}$ Instead, more comprehensive aberration correction is achieved when including a deformable mirror (DM) in the microscope such that depletion, excitation, and emission are simultaneously corrected. ${ }^{16}$ In both these two references, ${ }^{14,16}$ aberration correction was obtained by iteratively optimising the settings of the SLM or DM until a selected image quality metric is maximised. In this work we show how this approach can be readily extended to isoSTED, and we describe a novel image quality metric that is based on wavelet analysis. ${ }^{17}$

\section{ABERRATION CONTROL IN 4PI MICROSCOPY}

Aberration correction in isoSTED microscopy can be achieved by introducing a DM in the back focal plane of each of the two objectives comprising the $4 \mathrm{Pi}$ cavity. ${ }^{6}$ When operating the two DMs simultaneously, however, one needs to choose an arbitrary relative orientation between the pupils defined over the surface of each DM. An optimal choice was proposed in, ${ }^{10}$ and is depicted here in Fig. 1. By employing this arrangement, the Zernike coefficients ${ }^{18} \mathbf{a}$ of pupil $\Sigma_{1}$ and $\mathbf{b}$ of pupil $\Sigma_{2}$ can be easily related to a single vector of coefficients $\mathbf{c}$, which concisely describes the state of the aberration in the whole $4 \mathrm{Pi}$ cavity. In more detail, each element of $\mathbf{c}$ represents a certain coefficient $q_{i}^{s}$ - see Eq. (2) and Fig. (3) in, ${ }^{10}$ and Tab. 1 below. The aberration correction procedure can then be applied by optimising the values of the coefficients in vector $\mathbf{c}$ instead of alternating between optimising vectors $\mathbf{a}$ and $\mathbf{b}$ repeatedly.

Table 1. Mapping of some 4Pi aberration coefficients.

\begin{tabular}{|l|l|l|l|}
\hline $\mathbf{c}$ & $\mathbf{a}$ & $\mathbf{b}$ & notes \\
\hline$q_{1}^{+1}$ & $a_{1}=q_{1}^{+1}$ & $b_{1}=+q_{1}^{+1}$ & fringe shift \\
$q_{1}^{-1}$ & $a_{1}=q_{1}^{-1}$ & $b_{1}=-q_{1}^{-1}$ & piston \\
$q_{2}^{+1}$ & $a_{2}=q_{2}^{1}$ & $b_{2}=+q_{2}^{1}$ & covariant tip \\
$q_{3}^{+1}$ & $a_{3}=q_{3}^{1}$ & $b_{3}=+q_{3}^{1}$ & covariant tilt \\
$q_{4}^{+1}$ & $a_{4}=q_{4}^{1}$ & $b_{4}=+q_{4}^{1}$ & covariant defocus \\
$q_{2}^{-1}$ & $a_{2}=q_{2}^{1}$ & $b_{2}=-q_{2}^{1}$ & contra-variant tip \\
$q_{3}^{-1}$ & $a_{3}=q_{3}^{1}$ & $b_{3}=-q_{3}^{1}$ & contra-variant tilt \\
$q_{4}^{-1}$ & $a_{4}=q_{4}^{1}$ & $b_{4}=-q_{4}^{1}$ & contra-variant defocus \\
$\ldots$ & $\ldots$ & $\ldots$ & $\ldots$ \\
\hline
\end{tabular}

The mapping between coefficient $q_{i}^{s}$ and the corresponding non-zero coefficients in $\mathbf{a}$ and $\mathbf{b}$ is reported in Tab. 1. Here $i$ denotes the single index given by Noll for Zernike polynomials ${ }^{18}$ and $s$ indicates whether a coefficient is covariant $(s=+1)$ or contra-variant $(s=-1)$. The deformations of the foci created by the two objectives are overlapping for covariant coefficients, see Fig. (3) in. ${ }^{10}$ On the other hand, when contra-variant coefficients are applied, the foci undergo non-overlapping deformations. For a given $q_{i}^{s}$, the corresponding nonzero coefficients in pupil $\Sigma_{1}$ and $\Sigma_{2}$ are reported in the second and third columns. At this point it is relevant to emphasise which coefficients in $\mathbf{c}$ should be optimised by the aberration correction procedure. As pointed out in, ${ }^{10}$ piston coefficient $q_{1}^{-1}$ induces an aberration that does not affect the image quality and therefore should not be optimised. Similarly, displacement coefficients $q_{2}^{+1}, q_{3}^{+1}$, and $q_{4}^{+1}$ should be excluded, since they also do not affect the image quality but instead shift the position of the focus within the cavity. On the other hand, coefficient $q_{1}^{+1}$ does affect the image quality since it shifts the interference pattern at the focus along the optical axis. 
It should be noted that if the DMs are characterised ${ }^{19}$ externally, for example using an ad-hoc interferometer setup, or if the calibration provided by the manufacturer is used, then one can obtain the arrangement in Fig. 1 by flipping the $y$ axis of one of the two pupils. This is achieved by flipping the sign of all Zernike coefficients with odd index $i$ in one of the pupils, i.e., all coefficients having $\sin (\cdot)$-like azimuthal variation. Finally, it should be remarked that the calibration of the DMs must take into account the piston Zernike mode, which is commonly neglected in single-objective microscopes. This ensures correct simultaneous operation of the two DMs when applying the $q_{1}^{+1}$ and $q_{1}^{-1}$ coefficients. It also guarantees that controlling other coefficients does not result in undesired shifts of the interference pattern along the optical axis.

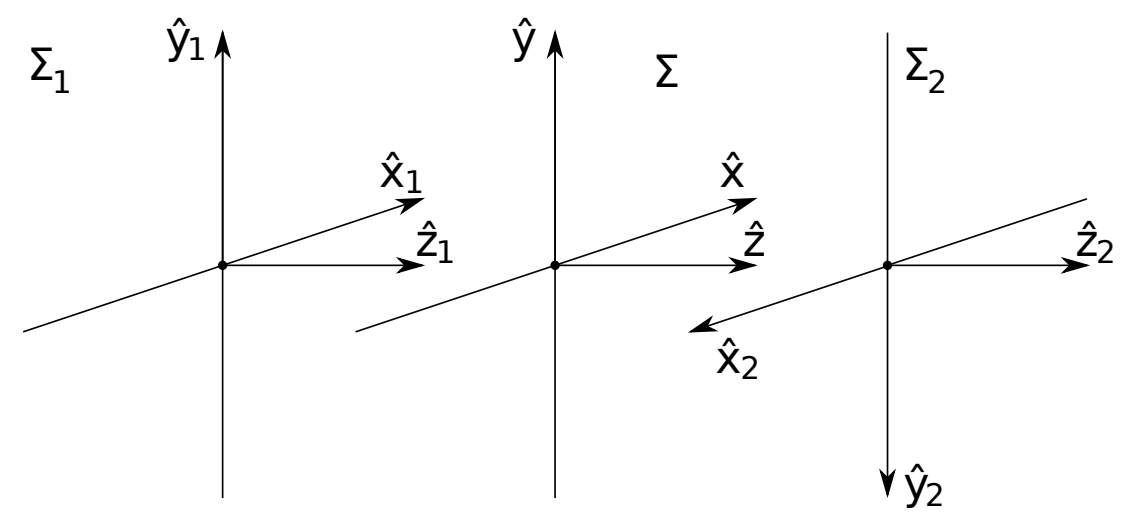

Figure 1. Arrangement of the reference frames within a $4 \mathrm{Pi}$ cavity. ${ }^{10}$ The reference frames for the pupils of the two objectives are denoted with $\Sigma_{1}$ and $\Sigma_{2}$. The reference frame for the common focal plane is indicated with $\Sigma$.

\section{DEFINITION OF THE IMAGE QUALITY METRIC}

In this section we introduce a novel image quality metric based on wavelet analysis. ${ }^{17}$ Previous efforts in applying aberration correction in single-objective STED ${ }^{14-16}$ aimed at detecting changes in the image quality using some form of Fourier analysis. For example, in ${ }^{14}$ a metric combining brightness and sharpness was selected, whereas in $^{16}$ a metric based on Fourier ring correlation ${ }^{20}$ (FRC) was considered. Nevertheless, a number of shortcomings are encountered while following this Fourier analysis approach. First, STED images have low photon counts and, as a result, are particularly affected by Poisson noise. This is especially the case for isoSTED microscopy, due to the increased depletion of the focal volume. Unfortunately, it is unclear how one may extend the image quality metrics mentioned above in order to implement optimal noise rejection strategies. In contrast, numerous Poisson noise rejection techniques have been developed using wavelets. ${ }^{21}$ Second, in general, the Fourier spectrum of the specimen under investigation has low high-frequency content, is asymmetric, and is not known a priori. As a result, when analysing the spectrum of recorded images, it is non-trivial to determine whether some high frequency contributions are truly due to the spectrum of the specimen or, instead, are merely due to noise. On the other hand, correcting aberrations that disrupt the super-resolution effect is of crucial importance and requires examining variations in the high frequency content of images. The time-frequency localisation capabilities of wavelets seem an ideal tool for this purpose. In this case, high spatial frequencies analysis can be localised to areas of the specimen that indeed exhibit fine features, and where the super-resolution effect is more noticeable. In contrast, high frequency coefficients determined using conventional Fourier analysis are not localised to certain areas of the specimen and therefore can be expected to be less sensitive to aberration.

Our starting point to establish a new image quality metric is the multi-scale variance stabilising transform ${ }^{21}$ (MS-VST), which is defined as

$$
\begin{aligned}
& a_{j}=h^{\uparrow j-1} * a_{j-1}, \\
& d_{j}=T_{j-1}\left(a_{j-1}\right)-T_{j}\left(a_{j}\right) .
\end{aligned}
$$

Here $a_{j}$ and $d_{j}$ are, respectively, the approximation and detail coefficients of the wavelet analysis at scale $j$. Given an input image $I$ of dimensions $N \times N$, one can let $a_{0}=I$ and subsequently compute the remaining coefficients $a_{j}$ 
and $d_{j}$ using the equations above. Note that each wavelet coefficient is in fact an image of dimensions $N \times N$ that captures both spatial and frequency information about the original image $I$. The discrete filter $h^{\uparrow j-1}$ and the transformation $T(\cdot)$ are defined in, ${ }^{21}$ where the authors also outline how Poisson noise rejection can be achieved by thresholding the detail coefficients $d_{j}$. An example of a MS-VST analysis is shown in Fig. 2.

(a) $I$

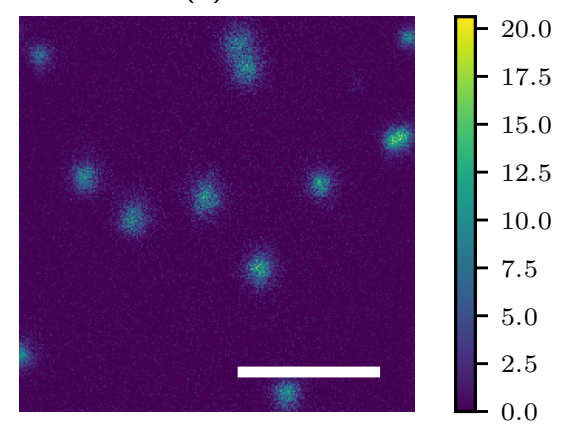

(c) $d_{3}$

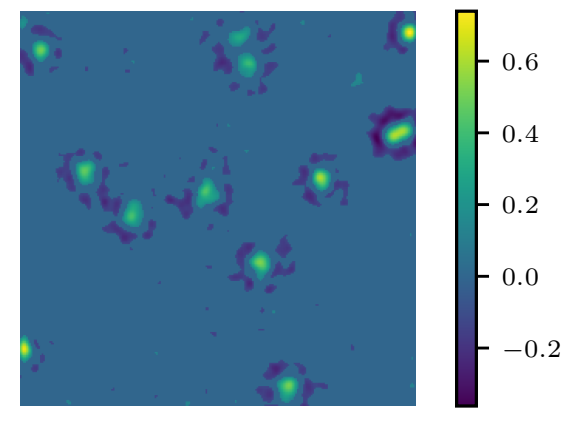

(b) $d_{2}$

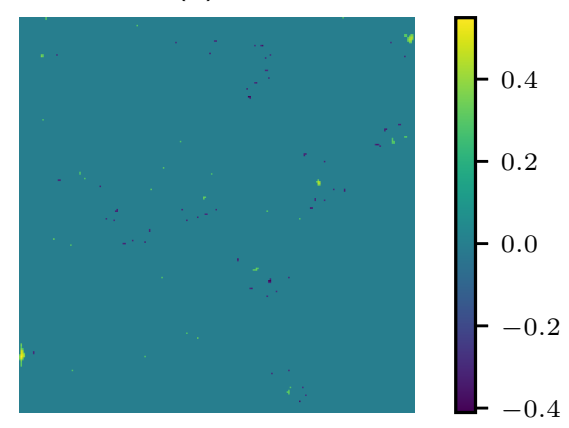

(d) $d_{4}$

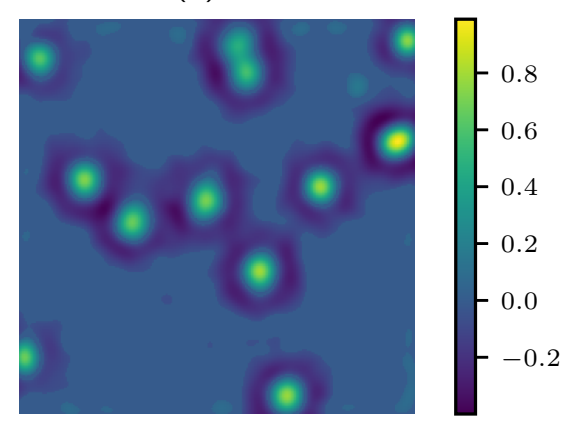

Figure 2. Wavelet analysis example. (a) raw image of fluorescent beads acquired with an isoSTED microscope. The image is $256 \times 256$ and the scale bar denotes $1 \mu \mathrm{m}$; (b)-(d) detail coefficients of the wavelet analysis at scales 2,3 , and 4 , respectively.

For the purposes of defining an image quality metric, we consider the detail coefficients $d_{j}$ and define a vector $\mathbf{m}$ as

$$
\mathbf{m}=\left[\begin{array}{c}
\sum_{k}\left|\left(d_{1}\right)_{k}\right|^{2} \\
\vdots \\
\sum_{k}\left|\left(d_{J}\right)_{k}\right|^{2}
\end{array}\right],
$$

where $\sum_{k}\left|\left(d_{j}\right)_{k}\right|^{2}$ denotes the sum of the $N^{2}$ elements $\left(d_{j}\right)_{k}$ of coefficient $d_{j}$, which are enumerated using a single index $k$ for simplicity. The first element of $\mathbf{m}$ is computed from $d_{1}$, i.e., the wavelet coefficient at scale $j=1$, and is sensitive to fine features appearing in $I$. The following elements of $\mathbf{m}$ correspond to larger scale indices $j$ and are sensitive to coarser features of $I$. As the amount of aberration affecting the microscope increases, fine features in the image $I$ become blurred and, as a consequence, the elements of $\mathbf{m}$ corresponding to small scales decrease in magnitude whereas the elements corresponding to larger scales increase. The aberration correction problem consists in inverting this trend by simultaneously optimising the elements of $\mathbf{m}$ as a function of the settings of the wavefront shaping device. We intend to report over the details about the optimisation of $\mathbf{m}$ in an upcoming publication. An example of the variation of $\mathbf{m}$ as a function of the aberration applied with the DMs is given in Fig. 3 . 

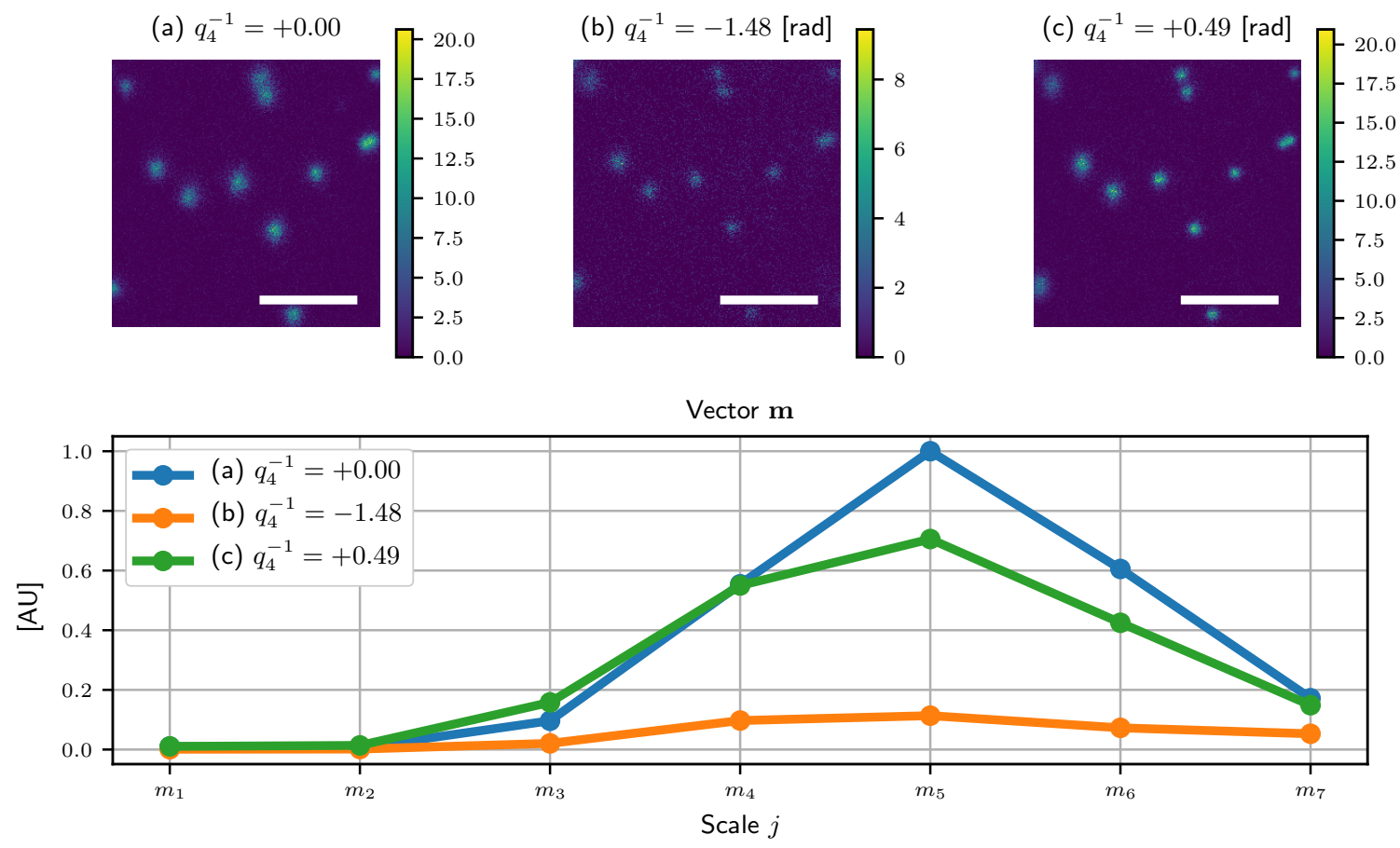

Figure 3. Variation of $\mathbf{m}$ as a function of the amount of aberration $q_{4}^{-1}$ applied with the DMs. (a) initial image acquired when $q_{4}^{-1}=0$ and initial state of $\mathbf{m}$ shown in blue; (b) applying a large amount of aberration results in a reduction of all elements of $\mathbf{m}$ shown in orange with respect to the initial state; (c) as aberration is removed and the resolution improves, a transfer of "energy" can be detected between the elements of $\mathbf{m}$ - compare the curve in green with the initial state in blue. Elements of $\mathbf{m}$ corresponding to small scales (e.g., $j=2,3$ ) increase whereas elements corresponding to large scales (e.g., $j=5,6,7)$ decrease markedly.

\section{CONCLUSIONS}

In this paper we discussed implementing adaptive optics in isoSTED microscopy. We first outlined how one can simultaneously control both deformable mirrors in a 4Pi cavity. Furthermore, we introduced a novel image quality metric that is based on wavelet analysis. This metric is particularly suited for low photon count images produced in isoSTED microscopy and is expected to outperform previously proposed aberration correction methods in single-objective STED microscopes.

\section{ACKNOWLEDGEMENTS}

The project is supported by the Wellcome Trust (095927/A/11/Z, 095927/B/11/Z, 203285/B/16/Z, 203285/C/16/Z), the G. Harold \& Leila Y. Mathers Foundation, the National Institutes of Health (P30 DK45735) and European Research Council (AdOMiS, no. 695140). J.B. discloses significant financial interest in Bruker Corp. and Hamamatsu.

\section{REFERENCES}

[1] Hell, S. W., "Microscopy and its focal switch," Nat. Methods 6(1), 24-32 (2009).

[2] Hell, S. and Stelzer, E. H. K., "Properties of a 4Pi confocal fluorescence microscope," J. Opt. Soc. Am. A 9(12), 2159-2166 (1992).

[3] Schmidt, R., Wurm, C. A., Jakobs, S., Engelhardt, J., Egner, A., and Hell, S. W., "Spherical nanosized focal spot unravels the interior of cells," Nat. Methods 5, 539-544 (2008). 
[4] Aquino, D., Schönle, A., Geisler, C., Middendorff, C. v., Wurm, C. A., Okamura, Y., Lang, T., Hell, S. W., and Egner, A., "Two-color nanoscopy of three-dimensional volumes by $4 \mathrm{Pi}$ detection of stochastically switched fluorophores," Nat. Methods 8, 353-359 (2011).

[5] Hao, X., Allgeyer, E. S., Booth, M. J., and Bewersdorf, J., "Point-spread function optimization in isoSTED nanoscopy," Opt. Lett. 40(15), 3627-3630 (2015).

[6] Huang, F., Sirinakis, G., Allgeyer, E., Schroeder, L., Duim, W., Kromann, E., Phan, T., Rivera-Molina, F., Myers, J., Irnov, I., Lessard, M., Zhang, Y., Handel, M., Jacobs-Wagner, C., Lusk, C., Rothman, J., Toomre, D., Booth, M., and Bewersdorf, J., "Ultra-high resolution 3D imaging of whole cells," Cell 166(4), 1028-1040 (2016).

[7] Böhm, U., Hell, S. W., and Schmidt, R., "4Pi-RESOLFT nanoscopy," Nat. Commun. 7, 10504 (2016).

[8] Deng, S., Liu, L., Cheng, Y., Li, R., and Xu, Z., "Effects of primary aberrations on the fluorescence depletion patterns of STED microscopy," Opt. Express 18(2), 1657-1666 (2010).

[9] Antonello, J., Kromann, E. B., Burke, D., Bewersdorf, J., and Booth, M. J., "Coma aberrations in combined two- and three-dimensional STED nanoscopy," Opt. Lett. 41(15), 3631-3634 (2016).

[10] Hao, X., Antonello, J., Allgeyer, E. S., Bewersdorf, J., and Booth, M. J., "Aberrations in 4Pi microscopy," Opt. Express 25(13), 14049-14058 (2017).

[11] Antonello, J., Burke, D., and Booth, M. J., "Aberrations in stimulated emission depletion (STED) microscopy," Opt. Commun. 404, 203-209 (2017).

[12] Booth, M. J., "Adaptive optics in microscopy," Philos. Trans. R. Soc. A 365(1861), 2829-2843 (2007).

[13] Booth, M. J., "Adaptive optical microscopy: the ongoing quest for a perfect image," Light Sci. Appl. 3, e165 (2014).

[14] Gould, T. J., Burke, D., Bewersdorf, J., and Booth, M. J., "Adaptive optics enables 3D STED microscopy in aberrating specimens," Opt. Express 20(19), 20998-21009 (2012).

[15] Gould, T. J., Kromann, E. B., Burke, D., Booth, M. J., and Bewersdorf, J., "Auto-aligning stimulated emission depletion microscope using adaptive optics," Opt. Lett. 38(11), 1860-1862 (2013).

[16] Patton, B. R., Burke, D., Owald, D., Gould, T. J., Bewersdorf, J., and Booth, M. J., "Three-dimensional STED microscopy of aberrating tissue using dual adaptive optics," Opt. Express 24(8), 8862-8876 (2016).

[17] Mallat, S., [A wavelet tour of signal processing], Academic press (1999).

[18] Noll, R. J., "Zernike polynomials and atmospheric turbulence," J. Opt. Soc. Am. 66(3), 207-211 (1976).

[19] Booth, M., Wilson, T., Sun, H.-B., Ota, T., and Kawata, S., "Methods for the characterization of deformable membrane mirrors," Appl. Opt. 44(24), 5131-5139 (2005).

[20] Nieuwenhuizen, R. P. J., Lidke, K. A., Bates, M., Puig, D. L., Grünwald, D., Stallinga, S., and Rieger, B., "Measuring image resolution in optical nanoscopy," Nat. Methods 10, 557-567 (2013).

[21] Zhang, B., Fadili, J. M., and Starck, J. L., "Wavelets, ridgelets, and curvelets for poisson noise removal," IEEE Trans. Image Process. 17(7), 1093-1108 (2008). 